\title{
Approach to Palmoplantar Pustulosis
}

\section{(1) Defne Özkoca, (1) Tuğba Kevser Uzunçakmak}

Istanbul University Cerrahpasa-Cerrahpasa Faculty of Medicine, Department of Dermatology, Istanbul, Turkey

\section{ABSTRACT}

Palmoplantar pustulosis is disease that manifests with grouped sterile pustules on the palms and soles. The disease manifests itself with recurrent grouped sterile pustules on a erythematous and keratotic basis, located on the palms and soles. The lesions may extend to the dorsum or the lateral aspects of the feet and hands. Various diseases can be considered in the differential diagnosis of the disease including pomphylox, dermatophyte infectios, scabies, insect bite, gonococcal infection, syphilis, palmoplantar psoriasis and acrodermatitis continua hallopeau. Treatment of the disease starts with general measures which are smoking cessation, use of emoillients and avoiding irritants. First line treatment modalities are topical corticosteroids, oral retinoids and phototherapy.

Keywords: Diagnosis, Palmoplantar pustulosis, Treatment

\section{Introduction}

Palmoplantar pustulosis is disease that manifests with grouped sterile pustules on the palms and soles. The inflammation is at the level of acrosyringium. In 1980, palmoplantar pustulosis was first described as a subtype of pustular psoriasis by Barber. However, in 2007, it was accepted as a seperate disease entity by the International Psoriasis Society [1].

Later the disease has been divided into two groups even though there is still ongoing discussion about the description of the disease. Disease type A was first reported by Andrews; the vesicles are preceded by pustules and it has a rare association with plaque type psoriasis. Disease type B was first reported by Barber, the pustules ocur without vesicles and it has a frequent association with plaque type psoriasis. The pustules are small in type $A$ whereas they are large in type $B$. Both subtypes have a female predominance and are associated with smoking [2].

\section{Clinical Features}

The disease manifests itself with recurrent grouped sterile pustules on a erythematous and keratotic basis, located on the palms and soles. The lesions may extend to the dorsum or the lateral aspects of the feet and hands. The lesions are painful, which decreases the quality of life of the patients. A $90 \%$ plaque psoriasis occurance rate has been reported in certain series. The lesions have a recurrent and chronic course, often treatment resistant. The third and fifth decades are the most common ages. The dermoscopy of the lesions show yellow structureless areas, which correspond to the pustules, dense interfollicular white desquamation and linearly arranged glomerular vessels. The nail findings that may be seen are onycholysis, pitting, splinter hemorrhages, subungal pustules and dystrophy [3].

In a study investigating the clinical characteristics of 48 palmoplantar pustulosis patients, a female predominance was observed: 33 females and 15 males. Seventy-two percent of the patients have a history of an 
average of 20 years pack/year cigarette use. Smoking was significantly more in male patients $(p=0.044)$. Forty-two percent of the patients had dental fillings, and of these patients two had positivity for nickel in patch testing. Six of the patients had occupational exposure history: 2 were cleaning staff, 2 were working at construction, 1 was hairdresser and 1 was tailor. None of the patients had a history of psoriasis; two of the patients had a family history of palmoplantar psoriasis. Seven of the patients presented with nail findings. Nine of the patients had musculoskeltal manifestations and four of the patients had autoimmune thyroiditis [4].

SAPHO syndrome is a syndrome related with palmoplantar pustulosis. Its components are synovitis, acne, palmoplantar pustulosis, hyperosteosis and osteitis. Palmoplantar pustulosis patients with musculoskeltal manifestations should be searched fort he SAPHO syndrome [5].

The known risk factors for palmoplantar pustulosis are female gender, smoking, autoimmun thyroiditis, allergic contact dermatitis due to nickel or other topical agents (via koebnerisation), upper respiratory tract infections (via autoreactivity), CARD14 and IL36RN mutations [2]. The disease severity increases as the year or number of packages cigarrette smoking increaes $(p=0.003)$. The severity of lesions was less in patients on ibuprofen treatment $(p<0.01)$. A significant relationship between comorbidities and lesion severity was not found [6].

As for the demographic factors, a study investigated the association of clinical and demographic factors with the severity of palmoplantar pustulosis in 203 patients. Severe disease was found to be associated with female gender, earlier disease presenatiton and smoking. The disease severity decreases dramatically with the cessation of smoking [7].

\section{Histopathology}

The histopathology of the disease shows epidermal sterile pustules, acanthosis, parakeratosis and inflammatory infiltrate [8].

\section{Differential Diagnosis}

The differential diagnoses of a patient applying to the clinic with palmoplantar pustules and vesicles are [9]:

- Pomphylox $\rightarrow$ contact to allergens, duration of symptoms, pruritus and or burning should be questioned.

- Dermatophyte infections (tinea pedis) $\rightarrow$ potassium hydroxide analysis should be performed.

- Scabies $\rightarrow$ should look for silion or tunnels, dermoscopy and skin scrapings should be performed.

- Insect bite $\rightarrow$ history of outdoor exposure should be questioned.

- Disseminated gonococcal infection $\rightarrow$ generalised lesions, fever and athralgia should be questioned.
- Second stage of syphylis $\rightarrow$ syphilitic chancre, other second stage lesions and history of suspicious intercourse should be questioned.

- Palmoplantar psoriasis $\rightarrow$ other psoriatic lesions should be examined.

- Acrodermatitis continua hallopeau $\rightarrow$ only one finger is involved.

\section{Treatment}

Treatment of the disease starts with general measures which are smoking cessation, use of emoillients and avoiding irritants. First line treatment modalities are topical corticosteroids, oral retinoids and phototherapy [9].

Topical corticosteroids is the safest and most commonly used treatment modality for palmoplantar pustulosis. Clobetasole propionate $(0.05 \%$ cream) or triamcinolone $(0.1 \%$ cream with occlusion) twice daily for one month is recommended as first line treatment. Topical anthralin, topical retinoids and topical calcipotriol can also be used even though they are not accepted as first line treatment modalities [9].

Systemic retinoids are the treatment of choice in patients who do not respond to topical treatment modalities. Acitretine can be initiated at a dose of $25 \mathrm{mg} /$ day and increased to $50 \mathrm{mg} /$ day in patients who tolerate the side effects. Three months of treatment is recommened and physicians should be watchful fort he possible retinoid side effects [10]. Alitretinoin is another systemic retinoid drug that can be used in the treatment of palmoplantar pustulosis. It was found to be therapeutically as efficient as acitretine, psoralene plus ultraviolet-A (PUVA) or acitretine plus PUVA [11].

Phototherapy is also useful in the treatment of palmoplantar pustulosis. Localised PUVA is the most commonly used phototherapy modality; three times weekly for at least 12 sessions. Narrow band ultraviolet-B, excimer laser and photodynamic treatment are also helpful. Retinoids plus PUVA is recommended as a second-line treatment [9].

Other treatment alternatives are oral tetracyclines, cyclosporine, metotreaxate, colchicine, itraconazole, tonsillectomy and diet deprived of gluten. The biologic treatment modalities are also considered in refractory cases; the previously reported biologics are ustekinumab [anti-interleukin (IL)-12/23], guselkumab (anti-IL-23), infliximab [anti-tumor necrosis factor (TNF)], etanercept (anti-TNF), adalimumab (anti-TNF), sekukinumab (anti-IL-17) and brodalumab (anti-IL-17) [9].

Guselkumab is a antibody drug that targets IL-23. T-helper 17 cells and IL-23 pathways are present in the pathogenesis of palmoplantar pustulosis. An 11 centered study in Japan investigated the use of guselkumab in the treatment of palmoplantar pustulosis. The patients were treated with $200 \mathrm{mg}$ subcutaneous injections on weeks 
0 and 4; and evaluated on weeks 16 and 24. Guselkumab was found to be effective in the treatment of palmoplantar pustulosis. The side effects were nasopharyngitis, headache and contact dermatitis [12]. Ustekinumab is an inhibitor of the p40 subunit of IL-12 and IL23 which have a role in the Th17 pathway as well. Nine patients received subcutaneous injections of ustekinumab $45 \mathrm{mg}$ on weeks 0 and 4 . Complete resolution of the lesions was observed on week 16. Ustekinumab is a safe and effective treatment modality in the treatment of palmoplantar pustulosis [13].

The inhibition of the Jannus kinase (JAK) pathway is an inhibitor of the IL-8 pathway. Previously, the inhibition of IL-8 was found to decrease the symptoms of plamoplantar pustulosis. A 45 years old female patient with refractory palmoplantar pustulosis lesions and psoriatic arthritis was treated with tofacitinib (JAK inhibitor, 10 $\mathrm{mg} /$ day, oral). The lesions subsided starting from the second week of treatment. Thus, tofacitinib is also a treatment alternative for palmoplantar pustulosis [14].

\section{Ethics}

Peer-review: Internally peer-reviewed.

\section{Authorship Contributions}

Concept: D.Ö., T.K.U., Design: D.Ö., T.K.U., Data Collection or Processing: D.Ö., T.K.U., Analysis or Interpretation: D.Ö., T.K.U., Literature Search: D.Ö., T.K.U., Writing: D.Ö., T.K.U.

Conflict of Interest: No conflict of interest was declared by the authors.

Financial Disclosure: The authors declared that this study received no financial support.

\section{References}

1. Raposo I, Torres T. Palmoplantar Psoriasis and Palmoplantar Pustulosis: Current Treatment and Future Prospects. Am J Clin Dermatol 2016;17:349358.
2. Murakami M, Terui T. Palmoplantar pustulosis: Current understanding of disease definition and pathomechanism. J Dermatol Sci 2020;98:13-19.

3. Oktem A, Uysal PI, Akdoğan N, Tokmak A, Yalcin B. Clinical characteristics and associations of palmoplantar pustulosis: an observational study. An Bras Dermatol 2020;95:15-19.

4. Oktem A, Uysal PI, Akdoğan N, Tokmak A, Yalcin B. Clinical characteristics and associations of palmoplantar pustulosis: an observational study. An Bras Dermatol 2020;95:15-19.

5. Hirosawa T, Kawamoto S, Shimizu T. SAPHO syndrome. BMJ Case Rep 2019;12:e233221.

6. Putra-Szczepaniak M, Reich A, Jankowska-Konsur A, Czarnecka A, BagłajOleszczuk M, Hryncewicz-Gwóźdź A. Pack-year cigarette smoking affects the course of palmoplantar pustulosis. Adv Clin Exp Med 2021;30:189-195.

7. Benzian-Olsson N, Dand N, Chaloner C, Bata-Csorgo Z, Borroni R, Burden AD, Cooper HL, Cornelius V, Cro S, Dasandi T, Griffiths CEM, Kingo K, Koks S, Lachmann H, McAteer H, Meynell F, Mrowietz U, Parslew R, Patel P, Pink AE, Reynolds NJ, Tanew A, Torz K, Trattner H, Wahie S, Warren RB, Wright A, Barker JN, Navarini AA, Smith CH, Capon F; ERASPEN consortium and the APRICOT and PLUM study team. Association of Clinical and Demographic Factors With the Severity of Palmoplantar Pustulosis. JAMA Dermatol 2020;156:1-8.

8. Masuda-Kuroki K, Murakami M, Kishibe M, Kobayashi S, Okubo Y, Yamamoto T, Terui T, Sayama K. Diagnostic histopathological features distinguishing palmoplantar pustulosis from pompholyx. J Dermatol 2019;46:399-408.

9. Misiak-Galazka M, Zozula J, Rudnicka L. Palmoplantar Pustulosis: Recent Advances in Etiopathogenesis and Emerging Treatments. Am J Clin Dermatol 2020;21:355-370.

10. Obeid G, Do G, Kirby L, Hughes C, Sbidian E, Le Cleach L. Interventions for chronic palmoplantar pustulosis. Cochrane Database Syst Rev 2020;1:CD011628.

11. Brunasso AMG, Massone C. Alitretinoin therapy for palmoplantar pustulosis. Br J Dermatol 2017;177:578-579.

12. Terui T, Kobayashi S, Okubo Y, Murakami M, Hirose K, Kubo H. Efficacy and Safety of Guselkumab, an Anti-interleukin 23 Monoclonal Antibody, for Palmoplantar Pustulosis: A Randomized Clinical Trial. JAMA Dermatol 2018;154:309-316.

13. Hegazy S, Konstantinou MP, Bulai Livideanu C, Tauber M, Uthurriague C, Paul C. Efficacy of ustekinumab in palmoplantar pustulosis. J Eur Acad Dermatol Venereol 2018;32:e204-e206.

14. Haynes D, Topham C, Hagstrom E, Greiling T. Tofacitinib for the treatment of recalcitrant palmoplantar pustulosis: A case report. Australas J Dermatol 2020;61:e108-e110. 